\begin{tabular}{|c|l|}
\hline Title & Generalized phase retrieval algorithm based on information measures \\
\hline Author(s) & Shioya, Hiroyuki; Gohara, Kazutoshi \\
\hline Citation & $\begin{array}{l}\text { Optics Communications, 266(1), 88-93 } \\
\text { https://doi.org/10.1016/.0ptcom.2006.04.034 }\end{array}$ \\
\hline Issue Date & 2006-10-01 \\
\hline Doc URL & http://hdl.handle.net/2115/14892 \\
\hline Type & article(author version) \\
\hline File Information & Oc266-1.pdf \\
\hline
\end{tabular}

Instructions for use 


\title{
Generalized Phase Retrieval Algorithm based on Information Measures
}

\author{
Hiroyuki Shioya $^{a}$ Kazutoshi Gohara ${ }^{\mathrm{b}}$ \\ ${ }^{a}$ Department of Computer Science and System Engineering, Muroran Institute of \\ Technology, Mizumoto, Muroran, 050-8585, Japan \\ ${ }^{\mathrm{b}}$ Department of Applied Physics, Graduate School of Engineering, Hokkaido \\ University, Sapporo, 060-8628, Japan
}

\begin{abstract}
An iterative phase retrieval algorithm based on the maximum entropy method (MEM) is presented. Introducing a new generalized information measure, we derive a novel class of algorithms which includes the conventionally used error reduction algorithm and a MEM-type iterative algorithm which is presented for the first time. These different phase retrieval methods are unified on the basis of the framework of information measures used in Information Theory.
\end{abstract}

Key words: Phase retrieval, Information measures PACS: 42.30.Rx, 89.70.+c

\section{Introduction}

An iterative Fourier phase retrieval method using intensity measurements for recovering the phase has been used in a diverse range of fields including electron microscopy, astronomy, crystallography, synchrotron X-ray and others. The first research into phase retrieval methods was the Gerchberg-Saxton algorithm based on iterative transformation back and forth between the object domain and the Fourier domain [1]. The process of replacing a prior object function obtained under the Fourier constraints has been performed including the error reduction (ER) method, the input and output (IO) method, and the hybrid input and output (HIO) method. Fienup (1982) has presented a comparison of these methods, clarifying in particular the relationship between the error reduction and the steepest descent method; this was the second important achievement of the research regarding phase retrieval methods [2]. In regard to the uniqueness of the phase problem, the oversampling ratio has been 
discussed in [3] and mathematical analyses of the problem have been presented in $[4,5]$. Recently, based on these iterative algorithms, advanced experiments of nano-scale imaging have progressed through the use of a synchrotron Xray having a coherent source and a transmission electron microscope (TEM) equipped with a field emission gun [10].

The maximum entropy method (MEM) used in the field of crystallography [1116] does not provide a clear representation of an iterative algorithm. However, the MEM can be effective for phase retrieval and bears some resemblance to phase retrieval methods involved in the analysis using the Fourier-domain constraint [17].

As a result, although computationally the phase retrieval algorithm has been fundamentally established, it remains necessary to select one of the various algorithms, or to develop an applicable combination of algorithms, understanding that uncertainty concerning the parameters will increase. Thus, the relationships among the various methods of phase retrieval need to be clarified for further experimentation.

In the present paper, we first derive an iterative MEM-type algorithm for phase retrieval. Second, we derive a new class of phase retrieval algorithms using a generalized information measure of $\gamma$-divergence. A collective view of well-known iterative algorithms for phase retrieval is given. As a result, the relationships among these various methods are clarified; specifically, the object update rule of the iterative process in phase retrieval is shown to be equivalent to the minimum pseudo-distance measure between two non-negative functions when single-intensity measurements of the Fourier domain are used.

\section{Phase Retrieval Algorithms and the Maximum Entropy Method}

\subsection{ER and $H I O$}

Phase retrieval is represented as a correlative diagram of the Fourier and inverse Fourier transforms between the object domain and the Fourier domain, i.e., the Gerchberg-Saxton iterative algorithm. Fienup's reconstruction of the phase process using the intensity measurements of both domains is as follows [2]: (i) Fourier transform the prior object $\rho$ into $F$; (ii) replace $F$ with $F^{\prime}$, whose amplitude is given by the experiment in the Fourier domain (The phase of $F^{\prime}$ is the same as that of $F$, and the replaced amplitude is the constraint in the Fourier domain.); (iii) inverse Fourier transform $F^{\prime}$ into $\rho^{\prime}$; and (iv) replace $\rho^{\prime}$ with $\rho$ using the constraints in the object domain. This iterative transformation back and forth between the object domain and the Fourier 
domain is shown in Fig. 1. Although the setting of the constraints in the object domain is very important for phase retrieval experiments, we focus on the mathematical framework of iterative algorithms.

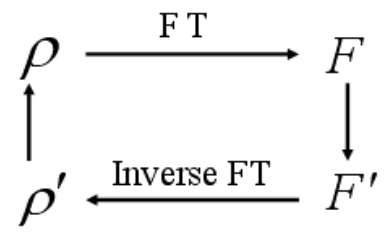

Fig. 1. Gerchberg-Saxton iterative diagram of phase retrieval.

In practical computation, we assume that both domains are 2-dimensional discrete spaces with squared arrays. Using the discrete Fourier transform, the $n$th iteration is given by the following equations:

$$
\begin{gathered}
F_{n}(\boldsymbol{k})=\sum_{\boldsymbol{r}} \rho_{n}(\boldsymbol{r}) \exp (-i 2 \pi \boldsymbol{k} \cdot \boldsymbol{r} / N), \\
\rho_{n}^{\prime}(\boldsymbol{r})=\frac{1}{N^{2}} \sum_{\boldsymbol{k}} F_{n}^{\prime}(\boldsymbol{k}) \exp (2 \pi i \boldsymbol{k} \cdot \boldsymbol{r} / N),
\end{gathered}
$$

where $\boldsymbol{r}=\left(r_{1}, r_{2}\right), \boldsymbol{k}=\left(k_{1}, k_{2}\right)$ and $r_{1}, r_{2}, k_{1}$, and $k_{2}=0, \cdots, N-1 . \rho_{n}$ is the object function of the $n$th iteration and $F_{n}$ is the Fourier transform of $\rho_{n} . F_{n}^{\prime}(\boldsymbol{k})=\left|F_{\text {obs }}(\boldsymbol{k})\right| \exp \left\{i \psi_{n}(\boldsymbol{k})\right\}, \psi_{n}(\boldsymbol{k})$ is the phase of $F_{n}(\boldsymbol{k})$, and $\left|F_{\text {obs }}(\boldsymbol{k})\right|$ is an intensity measurement in the Fourier domain. The object $\rho_{n}^{\prime}(\boldsymbol{r})$ is estimated by the inverse Fourier transform of $F_{n}^{\prime}(\boldsymbol{k})$. We restrict ourselves to the problem of recovering the phase in the Fourier domain using a single intensity measurement; moreover, we suppose that an object function is a real non-negative function. The ER is then described as

$$
\rho_{n+1}(\boldsymbol{r})=\left\{\begin{array}{ll}
\rho_{n}^{\prime}(\boldsymbol{r}) & \boldsymbol{r} \notin D \\
0 & \boldsymbol{r} \in D
\end{array},\right.
$$

where $D$ is the set of points at which $\rho_{n}^{\prime}$ violates the object-domain constraints. The following is the HIO, which is an improved version of the updating method with respect to the region breaking the object-domain constraints:

$$
\rho_{n+1}(\boldsymbol{r})=\left\{\begin{array}{ll}
\rho_{n}^{\prime}(\boldsymbol{r}) & \boldsymbol{r} \notin D \\
\rho_{n}(\boldsymbol{r})-\beta \rho_{n}^{\prime}(\boldsymbol{r}) & \boldsymbol{r} \in D
\end{array},\right.
$$

where $\beta$ is a positive constant. The ER and HIO are regarded as identical with respect to the updating at points satisfying the object-domain constraints. As mentioned above, phase retrieval is interpreted as the update with respect 
to the objective function using the constraints of the object domain and the Fourier domain. The MEM for structure analysis in crystallography utilizes the same type of renewal process of the object function. It is important to note the common framework of the two update procedures. Next, we derive a MEM-type algorithm as a new method for phase retrieval.

\section{2 $M E M$}

MEM has been applied to the estimation of crystal structures using X-ray diffraction data, and several representations of the formalism of MEM have been applied to Fourier inversion problems [13-16]. In the present work, we essentially follow the MEM formalism of Collins (1982) [11] (also see Sakata (1990) [12]). We describe the outline of the MEM for crystallography and derive a MEM-type algorithm for phase retrieval. The entropy expression $S$ is defined by Janes [18] as:

$$
\begin{array}{r}
S(\bar{\rho}, \bar{\tau})=-\sum_{\boldsymbol{r}} \bar{\rho}(\boldsymbol{r}) \ln \frac{\bar{\rho}(\boldsymbol{r})}{\bar{\tau}(\boldsymbol{r})}, \\
\bar{\rho}(\boldsymbol{r})=\frac{\rho(\boldsymbol{r})}{\sum_{\boldsymbol{r}^{\prime}} \rho\left(\boldsymbol{r}^{\prime}\right)}, \quad \bar{\tau}(\boldsymbol{r})=\frac{\tau(\boldsymbol{r})}{\sum_{\boldsymbol{r}^{\prime}} \tau\left(\boldsymbol{r}^{\prime}\right)},
\end{array}
$$

where $\rho(\boldsymbol{r})$ is an estimated function and $\tau(\boldsymbol{r})$ is a given prior function in the object domain. The object function corresponds to the electron density functions in crystallography. $\bar{\rho}(\boldsymbol{r})$ and $\bar{\tau}(\boldsymbol{r})$ denote the normalized density functions with respect to $\rho(\boldsymbol{r})$ and $\tau(\boldsymbol{r})$, respectively. We define the constraints concerning the known and unknown phases as follows:

$$
\begin{gathered}
C_{1}=\frac{1}{M_{1}} \sum_{\boldsymbol{k}} \frac{\left|F_{\text {cal }}(\boldsymbol{k})-F_{\text {obs }}(\boldsymbol{k})\right|^{2}}{\sigma^{2}(\boldsymbol{k})}, \\
C_{2}=\frac{1}{M_{2}} \sum_{\boldsymbol{k}} \frac{\left.|| F_{\text {cal }}(\boldsymbol{k})|-| F_{\text {obs }}(\boldsymbol{k})\right|^{2}}{\sigma^{2}(\boldsymbol{k})},
\end{gathered}
$$

where $M_{1}$ and $M_{2}$ are the number of reflections for known and unknown phases, respectively, and $F_{\text {cal }}$ is the calculated structure factor given as

$$
F_{\text {cal }}(\boldsymbol{k})=V \sum_{\boldsymbol{r}} \rho(\boldsymbol{r}) \exp (-i 2 \pi \boldsymbol{r} \cdot \boldsymbol{k}),
$$

where $V$ is the unit-cell volume, $F_{\text {obs }}$ is the observed structure factor, and $\sigma^{2}(\boldsymbol{k})$ is the variance of $F_{\text {obs }}(\boldsymbol{k})$. We use Lagrange's method while the expressed entropy is maximized under the constraint of the structure factor:

$$
L_{\lambda}=S(\rho, \tau)-\frac{\lambda_{1}}{2} C_{1}-\frac{\lambda_{2}}{2} C_{2}
$$


The maximum entropy probability is obtained as the following [11]:

$$
\begin{aligned}
\rho(\boldsymbol{r})= & \exp \left\{\ln \tau(\boldsymbol{r})+\frac{\lambda_{1} F_{0}}{M_{1}} \sum_{\boldsymbol{k}} \frac{\left(F_{\text {obs }}(\boldsymbol{k})-F_{\text {cal }}(\boldsymbol{k})\right)}{\sigma^{2}(\boldsymbol{k})} \exp \{-i 2 \pi \boldsymbol{k} \cdot \boldsymbol{r}\}\right. \\
& \left.+\frac{\lambda_{2} F_{0}}{M_{2}} \sum_{\boldsymbol{k}} \frac{\left(\left|F_{\mathrm{obs}}(\boldsymbol{k})\right| \exp [i \psi(\boldsymbol{k})]-F_{\text {cal }}(\boldsymbol{k})\right)}{\sigma^{2}(\boldsymbol{k})} \exp \{-i 2 \pi \boldsymbol{k} \cdot \boldsymbol{r}\}\right\},
\end{aligned}
$$

where $F_{0}$ is equal to the number of electrons in a unit cell. If $F_{\text {obs }}(\boldsymbol{k}), \sigma^{2}(\boldsymbol{k})$, $\lambda_{1}$ and $\lambda_{2}$ are given, we have an estimation of electron density function.

Next we derive an algorithm of phase retrieval based on the MEM, i.e., a MEMtype algorithm for phase retrieval using a single-intensity measurement. We introduce the integrated measure between two non-negative real functions as

$$
I(\rho, \tau)=\sum_{\boldsymbol{r}} \rho(\boldsymbol{r}) \ln \frac{\rho(\boldsymbol{r})}{\tau(\boldsymbol{r})}+\sum_{\boldsymbol{r}} \tau(\boldsymbol{r})-\sum_{\boldsymbol{r}} \rho(\boldsymbol{r}) .
$$

This measure is used for some statistical applications [19] and is an extension of $S(\bar{\rho}, \bar{\tau})$. All the information of the phase is unknown, so the Fourier constraints using the structure factors are given as

$$
R_{\mathrm{ph}}=\frac{1}{N^{2}} \sum_{\boldsymbol{k}}|| F_{\mathrm{cal}}(\boldsymbol{k})|-| F_{\mathrm{obs}}(\boldsymbol{k})||^{2}
$$

where $\left|F_{\text {obs }}\right|$ is an intensity measurement in the Fourier domain, and $F_{\text {cal }}$ is equal to the Fourier transform of an estimated object function $\rho$. Using the property of the least squares of the Fourier domain for phase retrieval [2], we have

$$
\frac{\partial}{\partial \rho} \sum_{\boldsymbol{k}}\left|F_{\text {cal }}(\boldsymbol{k})-F_{\text {obs }}(\boldsymbol{k})\right|^{2}=2\left(\rho(\boldsymbol{r})-\rho^{\prime}(\boldsymbol{r})\right),
$$

where $\rho^{\prime}(\boldsymbol{r})$ is the inverse Fourier transform of $\left|F_{\text {obs }}(\boldsymbol{k})\right| \exp [i \psi(\boldsymbol{k})]$, and $\psi(\boldsymbol{k})$ is the phase used in the Fourier-domain constraint of phase retrieval, i.e., $\psi(\boldsymbol{k})$ is a phase of $F_{\text {cal }}$. Using Lagrange's method for the minimum of $I(\rho, \tau)+\frac{\lambda}{2} R_{\mathrm{ph}}$, we obtain

$$
\rho(\boldsymbol{r})=\exp \left[\ln \tau(\boldsymbol{r})+\frac{\lambda}{N^{2}}\left(\rho^{\prime}(\boldsymbol{r})-\rho(\boldsymbol{r})\right)\right] .
$$

We use the assumption that $\rho$ is close to $\tau$ (the norm $|\rho-\tau|$ is sufficiently small). We then derive an update rule of the object function with respect to Eq. (15) by using the following settings: $\rho(\boldsymbol{r})$ and $\tau(\boldsymbol{r})$ in the right side of the equation refer to the prior object $\rho_{n}(\boldsymbol{r})$, and $\rho(\boldsymbol{r})$ in the left side of the equation is an updated object $\rho_{n+1}(\boldsymbol{r}) ; \psi(\boldsymbol{k})\left(=\psi_{n}(\boldsymbol{k})\right)$ is the phase of $F_{n}(\boldsymbol{k})$ given by the Fourier transform of $\rho_{n}(\boldsymbol{r})$ for the $n$th iteration. Then we have

$$
\rho_{n+1}(\boldsymbol{r})=\rho_{n}(\boldsymbol{r}) \exp \left[\xi\left(\rho_{n}^{\prime}(\boldsymbol{r})-\rho_{n}(\boldsymbol{r})\right)\right],
$$


where $\rho_{n}^{\prime}(\boldsymbol{r})$ is the object obtained by the inverse Fourier transform of $\left|F_{\text {obs }}\right| \exp \left(i \psi_{n}\right)$, and $\xi$ is a positive constant.

The variance $\sigma(\boldsymbol{k})$ is used in Eq. (11); however, we assume that such an uncertain factor is involved in $\left|F_{\text {obs }}(\boldsymbol{k})\right|$. Needless to say, this iterative formula can be derived using Eq. (13). The updating at which $\rho_{n+1}$ violates the objectdomain constraint is accomplished by the usual adaptive usage of the ER or the HIO.

\section{Generalized Algorithm for Phase Retrieval}

The expressed entropy $S$ used in the MEM denotes the relationship between two probability distributions in terms of information for discrimination. In Information Theory, a distance-like measure is used. The negative formula of $S$ is equivalent to an information measure known as the Kullback-Leibler divergence [20]:

$$
D_{k}(p, q)=\sum_{x \in \boldsymbol{X}} p(x) \ln \frac{p(x)}{q(x)}
$$

where $p$ and $q$ are probability distributions defined for the set $\boldsymbol{X}$, i.e., $\sum_{x \in \boldsymbol{X}} p(x)=$ $1, \sum_{x \in \boldsymbol{X}} q(x)=1, p(x) \geq 0$ and $q(x) \geq 0$ for any $x$ in $\boldsymbol{X}$. The term "information divergence" is often used in Information Theory [21,22]. The inequality $D_{k}(p, q) \geq 0$ is always satisfied for any $p, q$, and its equality is established if and only if $p(x)=q(x)$ for any $x$ in $\boldsymbol{X}$.

As mentioned above, the X-ray diffraction problem using the MEM is the minimization of the Kullback-Leibler divergence under the constraint of feasible information at the Fourier domain. Although there are several methods for phase retrieval, including the HIO, ER, and MEM, it is important in the use and development of an iterative algorithm of phase retrieval to clarify the theoretical relationships among these methods.

Here, we use a generalization process to obtain an information divergence measure between two probability distributions following the formalism of [23]. We introduce the following f-divergence [24]:

$$
D_{f}(p, q)=\sum_{x} p(x) f\left(\frac{q(x)}{p(x)}\right)
$$

where $f$ is a convex function defined on the open interval $(0, \infty)$ that satisfies $f(1)=0$ and is strictly convex at $u=1 . D_{f}(p, q) \geq 0$ is satisfied and its equality is established if and only if $p(x)=q(x)$ for any $x$ in $\boldsymbol{X}$. By applying various convex functions to $f$ in Eq. (18), several well-known information divergences are obtained. If $f(u)=-\ln u, D_{f}$ becomes the Kullback-Leibler 
divergence. The following is known as an $\alpha$-divergence [25]:

$$
D_{\alpha}(p, q)=\frac{1}{\alpha(1-\alpha)} \sum_{x}\left\{(1-\alpha) p(x)+\alpha q(x)-p(x)^{1-a} q(x)^{\alpha}\right\},
$$

where $\alpha$ is a real number. This is a kind of $f$-divergence, because of the setting of $f$ in Eq. (18):

$$
f(u)=\frac{1}{\alpha(1-\alpha)}\left\{1-u^{\alpha}+\alpha(u-1)\right\}
$$

The original formulation of $\alpha$-divergence is known as Renyi's order $\alpha$ information gain [26]; moreover, this formulation is closely related to Tsallis entropy [27]. By using the substitutions $p, q \rightarrow p^{s}, q^{s}$ (where $s$ is a real number), $s=1+\gamma$ and $\alpha=\gamma /(1+\gamma)$, and excluding the constant, we have

$$
D_{\gamma, 1+\gamma}(p, q)=\sum_{x}\left(\frac{1}{\gamma} p(x)\left(p(x)^{\gamma}-q(x)^{\gamma}\right)-\frac{1}{1+\gamma}\left(p(x)^{1+\gamma}-q(x)^{1+\gamma}\right)\right) .
$$

The above-clarified derivation process, i.e., the genealogy from $f$-divergence, has been clearly presented in a previous work [23]. $D_{\gamma, 1+\gamma}(p, q)$ is equal to $I(p, q)$ (Eq. (12)) for the case in which $\gamma \rightarrow 0$. The present integrated measure, which is called $(\gamma, 1+\gamma)$-divergence ( $\gamma$-divergence in short) in [23], has a number of statistical applications $[28,29]$. It is important to note that $\gamma$ divergence is a discriminant measure between two non-negative functions with finite volume. Thus, concerning the MEM-type iterative algorithm in Eq. (16), the normalization process, Eq. (5), is not needed.

The procedure for calculating the maximum entropy distribution is an update of the object function $\rho$ using the feasible information in the Fourier domain. The phase of the Fourier domain is not given, so we use a structure factor with unknown phase. The Fourier constraints using structure factors is the same as Eq. (13)

$$
R_{\mathrm{ph}}=\frac{1}{N^{2}} \sum_{\boldsymbol{k}}|| F_{\text {cal }}(\boldsymbol{k})|-| F_{\text {obs }}(\boldsymbol{k})||^{2}
$$

Equation (22) and $\gamma$-divergence give the following minimization problem:

$$
L_{\gamma}=D_{\gamma}(\rho, \tau)+C_{\gamma} R_{\mathrm{ph}}
$$

The first term of the object function indicates the $\gamma$-divergence from the prior object to the obtained object by the update rule, and the second term indicates the Fourier constraints. The positive constant $C_{\gamma}$ shows the mixture rate of the feasible information in the Fourier domain.

Using the property of the least squares of the Fourier domain [2] as well as 
Eq. (14), we have

$$
\frac{\partial}{\partial \rho} \sum_{\boldsymbol{k}}\left|F_{\text {cal }}(\boldsymbol{k})-F_{\text {obs }}(\boldsymbol{k})\right|^{2}=2\left(\rho(\boldsymbol{r})-\rho^{\prime}(\boldsymbol{r})\right)
$$

where $\rho^{\prime}(\boldsymbol{r})$ is the inverse Fourier transform of $\left|F_{\text {obs }}(\boldsymbol{k})\right| \exp [i \psi(\boldsymbol{k})]$, and $\psi(\boldsymbol{k})$ is the phase used in the Fourier-domain constraint of phase retrieval. The setting $\frac{\partial}{\partial \rho} L_{\gamma}=0$ gives

$$
\frac{\rho(\boldsymbol{r})^{\gamma}-1}{\gamma}=\frac{\tau(\boldsymbol{r})^{\gamma}-1}{\gamma}+C_{\gamma}\left(\rho^{\prime}(\boldsymbol{r})-\rho(\boldsymbol{r})\right)
$$

When $\rho$ is close to $\tau$ (the norm $|\rho-\tau|$ is sufficiently small), we can derive an update rule of the object function with respect to the above minimization using the same method used to derive Eq. (16). We use the following settings: $\rho(\boldsymbol{r})$ and $\tau(\boldsymbol{r})$ on the right side of Eq. (25) refer to the prior object $\rho_{n}(\boldsymbol{r})$, and $\rho(\boldsymbol{r})$ on the left side of the equation is the updated object $\rho_{n+1}(\boldsymbol{r}) ; \psi(\boldsymbol{k})\left(=\psi_{n}(\boldsymbol{k})\right)$ is the phase of $F_{n}(\boldsymbol{k})$ given by the Fourier transform of $\rho_{n}(\boldsymbol{r})$ for the $n$th iteration. We then have the following update rule:

$$
\rho_{n+1}(\boldsymbol{r})=\left\{\rho_{n}(\boldsymbol{r})^{\gamma}+\gamma C_{\gamma}\left(\rho_{n}^{\prime}(\boldsymbol{r})-\rho_{n}(\boldsymbol{r})\right)\right\}^{\frac{1}{\gamma}}
$$

where $\rho_{n}^{\prime}(\boldsymbol{r})$ is the object obtained by the inverse Fourier transform of $\left|F_{\text {obs }}\right| \exp \left(i \psi_{n}\right)$. A constraint for which $\gamma \geq 0$ is required because $\rho(\boldsymbol{r})=0$ for some $\boldsymbol{r}$. A setting of $\gamma \rightarrow 0$ gives

$$
\rho_{n+1}(\boldsymbol{r})=\rho_{n}(\boldsymbol{r}) \exp \left[C_{0}\left(\rho_{n}^{\prime}(\boldsymbol{r})-\rho_{n}(\boldsymbol{r})\right)\right]
$$

which is the same as Eq. (16). A setting of $\gamma \rightarrow 1$ gives

$$
\rho_{n+1}(\boldsymbol{r})=\left(1-C_{1}\right) \rho_{n}(\boldsymbol{r})+C_{1} \rho_{n}^{\prime}(\boldsymbol{r}) .
$$

In the case of $\gamma \rightarrow 0$, the update rule of Eq. (27) is equivalent to the MEMtype algorithm of Eq. (16). In the case in which $\gamma=1$, the update rule of Eq. (28) has the same representation as the ER, and the constant $C_{1}$ indicates the mixture rate of the feasible information at the object domain. The relationship between the MEM and ER based on $\gamma$-divergence is shown in Fig. 2. Any $\gamma$ in $[0,1]$ can be used. This figure represents the family of update methods parameterized by $\gamma$, with both the MEM and ER being including in the family. The HIO is the same as the ER except for the update rule at the region breaking the object-domain constraints. Therefore, the HIO is included in the family. 
MEM ER

$$
\gamma=0 \quad \gamma=1
$$

Fig. 2. The relationship between MEM and ER based on $\gamma$-divergence.

\section{Numerical example}

We provide a simple numerical example using Eq. (26). The setting is as follows. The target object is a CNT-like object, which is defined on the squared array domain $(256 \times 256$ pixels $)$. A real positive constraint is given and a sufficient support area is given as the object-domain constraint. The oversampling ratio (= total area / support area) is 6. For the updating method with respect to the region breaking the object-domain constraints, Eq. (3) is used. Concerning the value of $\gamma, 11$ cases $(\gamma=0,0.1, \cdots, 0.9,1)$ are used. The MEM and ER correspond to the cases $\gamma=0$ and $\gamma=1$, respectively. The coefficient $C_{\gamma}$ is 0.01 for every $\gamma$. A random object is used as an initial object function for all cases. In order to demonstrate whether or not phase retrieval is established, we define the $\mathrm{R}$ factor for the $n$th iteration as

$$
\text { R factor }=\frac{\sum_{\boldsymbol{k}}|| F_{n}(\boldsymbol{k})|-| F_{\text {obs }}(\boldsymbol{k})||}{\sum_{\boldsymbol{k}}\left|F_{\mathrm{obs}}(\boldsymbol{k})\right|} .
$$

The figures (a), (b), and (c) in Fig. 3 are the objects obtained by the MEMtype algorithm $(\gamma=0)$ with 20,300, and 1000 iterations, respectively. The final image (c) is almost the same as the target image. The graph shows the transitions of the $\mathrm{R}$ factor. The convergence of each transition denotes that an appropriate estimation is obtained for every $\gamma$.

\section{Discussion}

An iterative phase retrieval algorithm was discussed on the basis of the GerchbergSaxton algorithm [1] and the steepest descent analysis presented by J. Fienup [2]. The MEM is related to the phase retrieval problem as a restriction of the phase in the Fourier-domain. The variance $\sigma^{2}(\boldsymbol{k})$ of the observed structure factor $F_{\text {obs }}$ has been introduced in the non-iterative MEM formula represented by Eq. (11). By introducing the Fourier constraints $R_{\mathrm{ph}}$ without the variance, a MEM-type iterative algorithm can be derived. The MEM is generally regarded as the minimization of Kullback-Leibler divergence with respect to the Fourier 


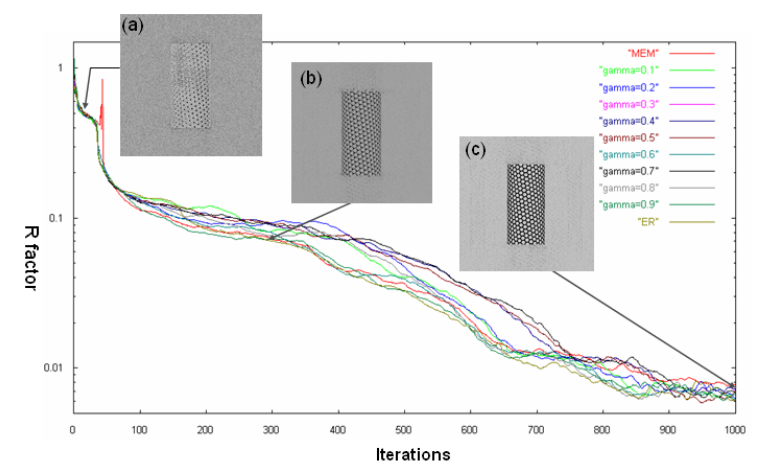

Fig. 3. ( Color ) The graph presents the transitions of the $\mathrm{R}$ factor using the cases in which $\gamma=0,0.1, \cdots, 0.9,1$. The figures (a), (b), and (c) are the objects obtained by the MEM-type algorithm $(\gamma=0)$ with 20,300, and 1000 iterations, respectively.

constraints; we derive a generalized update rule for estimating an object function using the $\gamma$-divergence measure. As a result, our new proposed update rule represents a simplified version of MEM and ER by setting the parameter of the $\gamma$-divergence. The constant $C_{\gamma}$ indicates the mixture rate of the feasible information in the Fourier-domain; hence, in the case in which $C_{\gamma}>0$ (there are some constraints of $\gamma$, for example $0<C_{0}<1$ ), our generalized update rule is almost entirely effective except for in the local minimum problem.

We demonstrate that a novel class of algorithms provides various different processes up to phase retrieval by a simple numerical example using a CNT-like object. The algorithm Eq. (26) is established if the prior and posterior object are close to each other. An iterative usage of the algorithm gives convergence in our simple numerical example. The global solution using the algorithm will be examined in future works. By using various settings of the coefficient $C_{\gamma}$ and the object-domain constraints, we confirmed various different transitions of the $\mathrm{R}$ factor. Thus, an appropriate setting of the parameter $\gamma$ of Eq. (26) and the object-domain constraints brings about the convergence. The refinement of the algorithms, especially the tuning of parameters $\gamma$ and $C_{\gamma}$, will be examined. The HIO is an effective update rule for the region breaking the object-domain constraints. Thus, concerning the update rule for such a region, a generalized iterative phase retrieval algorithm based on information measures remains unclear and needs to be examined from the view of Information Theory. Moreover, an extension of Information Theory based on a complex object function will be an important focus of our future research. Indeed, the importance of such theoretical research has been pointed out in [30]. Although some work remains, a profound theoretical understanding is indispensable for the further development of experiments on structure analysis using the iterative Fourier phase retrieval method. 


\section{Acknowledgments}

The authors would like to thank M. Sakata for the preliminary results related to the MEM-type algorithm in Eq. (16), which was derived for the first time in this study. We also thank T. Ishikawa and his research group for information regarding the recent advanced experiments using the phase retrieval algorithm in the X-ray field.

\section{References}

[1] R. W. Gerchberg and W. O. Saxton, Optic, 35,(1972) 237.

[2] J. R. Fienup, Applied Optics, 21, (1982) 2758.

[3] J. Miao, D. Sayre and H.N. Chapman, J. Opt. Soc. Am. A. A 15, (1998) 1662.

[4] Yu. M. Bruck and L. G Sodin, Opt. Commun. 30, 304 (1979)

[5] R. Barakat and G. Newsam, J. Math. Phys. 25, 2190 (1984)

[6] J. Miao, P. Charalambous, J. Kirz and D. Sayre, Nature, 400, (1999) 342.

[7] J. Miao, T. Ishikawa, B. Johnson, E.H. Anderson, B. Lai and K.O. Hodgson, Phys. Rev. Lett., 89, 088303 (2002).

[8] J. Miao, T. Ishikawa, E.H. Anderson and K.O. Hodgson, Phys. Rev. B 67, 174104 (2003).

[9] Y. Nishino, J. Miao and T. Ishikawa, Phys. Rev. B 68, 220101(R) (2003).

[10] J.M. Zuo, I. Vartanyants, M. Gao, R. Zhang, and L.A. Nagahara, Science 300, 1419 (2003).

[11] D.M. Collins, Nature (London), 298, 49 (1982).

[12] M. Sakata and M. Sato, Acta Cryst. A46, (1990) 63.

[13] A. Podjarny, D. Moras, J. Navaza, and P.M. Alizari, Acta Cryst. A44, (1988) 545.

[14] O.E. Piro, Acta Cryst., A39, (1983) 61.

[15] W. Wei, J. Apply. Phys. 18, (1985) 442.

[16] S.F. Gull and G.J. Daniel, Acta Cryst., A43, (1987) 112.

[17] M. Sakata, private communication.

[18] E. T. Jaynes, IEEE Trans. Syst. Cybern. SSC4, (1968) 227.

[19] I. Csiszár, Ann. Statist., 19, 4, (1991) 2032 . 
[20] S. Kullback and R.A. Leibler, Ann. Math. Stat., 22, (1951) 79.

[21] C.E. Shannon, A Mathematical Theory of Communication, Bell Sys. Tech. Journal, (1948) 27.

[22] T. Cover and J. A. Thomas, Elements of Information Theory, John Wiley \& Sons, New York 1991.

[23] M. Uchida and H. Shioya, IEICE, J87-A, 4, (2004) 546. [Electronics and Communication in Japan: Part 3, Fundamental Electronic Science, 88, 4, (2005) $35]$.

[24] I. Csiszár, Studia Sci. Math. Hunger., 2, (1967) 299 .

[25] A. Amari and H. Nagaoka, Methods of Information Geometry, AMS Oxford, 191, (2000).

[26] A. Renyi, in Proc. of the Fourth Berkeley Symposium on Mathematical Statistics and Probability, Berkeley, 1, (1961) 541.

[27] C. Tsallis, J. Stat. Phys. 52, (1988) 479.

[28] A. Basu, I. R. Harris, N. Hjort and M. C. Jones, Biometrika, 85, (1998) 549.

[29] M. Minami and S. Eguchi, Neural Computation, 14, 8, (2002) 1859.

[30] V. Elser, J. Opt. Soc. Am. A. 20, (2003) 40. 